\section{Impacted Right Maxillary Third Molar Fused With Supernu- merary Tooth}

\section{Sir,}

An 18-year male presented to us for examination of oral cavity. The patient was physically healthy without drug allergy or hereditary disease. Cone beam computed tomograph (CBCT) showed the presence of a supernumerary tooth fused to impacted right maxillary third molar (Figures 1 and 2). The third molar was impacted vertically in the right maxillary bone. It was shown that the proximal middle root of the supernumerary tooth was completely fused with the third molar distal root, and crown was separated from the third molar at an angle. The molar was fused with the supernumerary tooth in maxillary bone, adjacent to the maxillary sinus and seemed to be good by imaging examination. The patient had no intention of treatment. He was recommended to be examined and followed up once a year. Oral hygiene instruction was conducted; and the patient was suggested to visit, if any symptomoccurs.

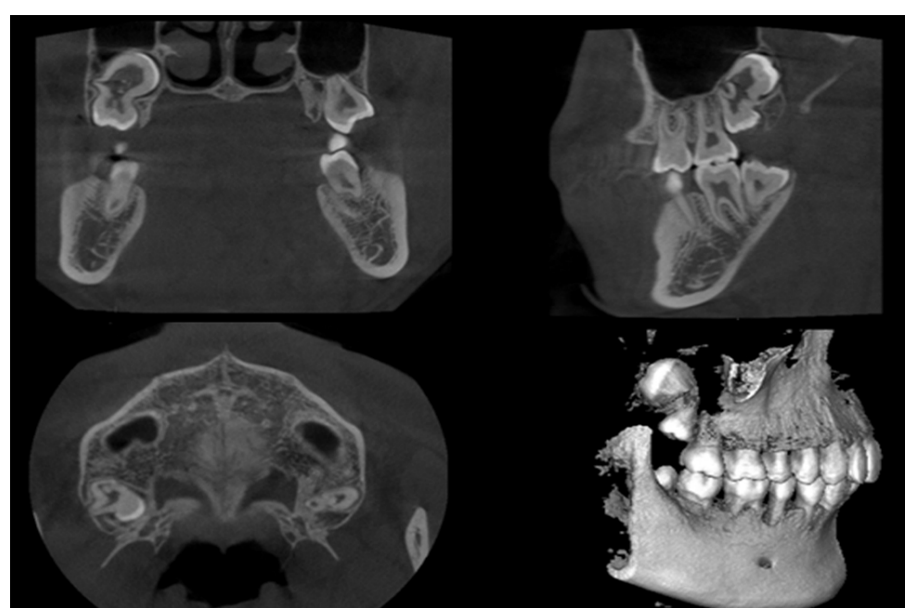

Figure 1: Cone beam computed tomograph (CBCT) imaging: The coronal, sagittal, andaxial planes, and three-dimensional reconstruction of supernumerary tooth fused to impacted right maxillary third molar.

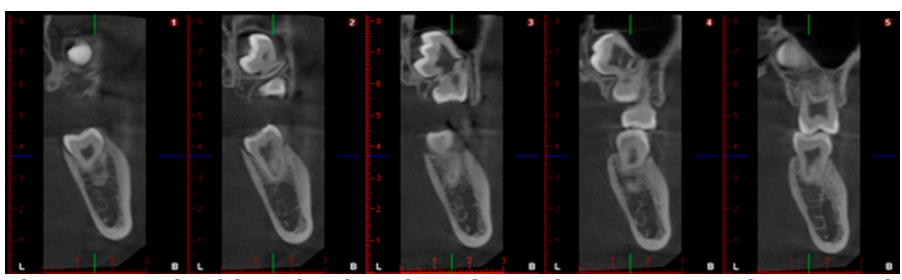

Figure 2: Sagittal layering imaging of cone beam computed tomograph (CBCT): There were five layers of sagittal imaging of supernumerary tooth fused to impacted right maxillary third molar, and each layer was 3 centimeters wide.

Fusion is a combination of two different tooth buds at any stage of dental development; and is rarely seen in permanent teeth. ${ }^{1}$ Teeth are connected to canals, pulp chambers, and dentine in the different development stages. It is related to the epithelial and mesenchymal germ layers, leading to abnormal teeth morpho-logy. Genes, race, stress, or physical force interacting with developing tooth buds and trauma are thought to be related to tooth fusion. ${ }^{1}$ The supernumerary tooth is an additional entity to the normal series of teeth that erupts or remains un-erupted in primary and permanent teeth. It represents a unique developmental anomaly of pattern and morphogenesis and may be single or multiple in the premaxilla. Incidence of supernumerary teeth has been reported as $1.5 \%$. It was more common in males than in females with a mean age of $14.13 \pm 5.6$ years. $^{2}$

Several associated diseases include caries, gingival inflammation, irreversible pulpitis with secondary periapical involvement, pulpitis and apical periodontitis, and paradental cyst..$^{3-5}$ Diagnosis of the third molar fused with the supernumerary tooth is based on the clinical history and radiographic detection. The condition needs to be evaluated by dental and imaging examination. Most such teeth are removed as a result of complicated anatomic structure, teeth location, and difficulty in rubber dam isolation. If endodontic therapy is contemplated, regularfollow-up is needed.

\section{CONFLICT OF INTEREST:}

Authors declared no conflict of interest.

\section{AUTHORS' CONTRIBUTION:}

XC: Collected data, reviewed literature, drafted figures and manuscript.

LC, KC: Drafted the manuscript and collected data.

\section{REFERENCES}

1. Zeylabi A, Shirani F, Heidari F, Farhad AR. Endodontic management of a fused mandibular third molar and distomolar: A case report. Aust Endod J 2010; 36:29-31.

2. Pippi R. Odontomas and supernumerary teeth: Is there a common origin? Int J Med Sci 2014; 11:1282-97.

3. Chen RJ, Yang JF. Fusion of a third molar with an invaginated supernumerary molar. Oral Surg Oral Med Oral Pathol 1990; 70:526-7.

4. Turell IL, Zmener O. Endodontic management of a mandibular third molar fused with a fourth molar. Int Endod J 1999; 32: 229-31.

5. Prakash AR, Reddy PS, Rajanikanth M. Paradental cyst associated with supernumerary tooth fused with third molar: A rare case report. J Oral Maxillofac Pathol 2012; 16:131-3.

Xinjia Cai ${ }^{1}$, Lin Cui ${ }^{1}$ and Kejia Chen ${ }^{2}$

${ }^{1}$ Department of Oral Pathology, Xiangya Stomatological Hospital, Central South University, Hunan, China

${ }^{2}$ Department of Oral Radiology, Xiangya Stomatological Hospital, Central South University, Hunan, China

Correspondence to: Kejia Chen, Department of Oral Radiology, Xiangya Stomatological Hospital, Central South University, Hunan, China

E-mail:29326625@qq.com

Received: September 18, 2019; Revised: September 18, 2019;

Accepted: September 22, 2019

DOI: https://doi.org/10.29271/jcpsp.2020.05.552 